\title{
Triploidy and other chromosomal abnormalities in a selected line of chickens
}

\author{
MH Thorne, RK Collins, BL Sheldon \\ CSIRO Division of Animal Production, Poultry Genetics, \\ PO Box 184, North Ryde, NSW 2113, Australia
}

(Proceedings of the 9th European Colloquium on Cytogenetics of Domestic Animals; Toulouse-Auzeville, 10-13 July 1990)

triploid / chicken / meiosis / diploid ova

Triploidy has been reported in the chicken in both embryos and adults (de Boer et al, 1984). Triploid chick embryos with ZWW sex chromosomes are not viable beyond the first few days of incubation, but some with ZZW and ZZZ sex chromosomes survive to hatching and maturity. The $\mathrm{ZZW}$ triploids have an adult intersex phenotype and ZZZ triploids a male phenotype. Both are sterile (Thorne et al, 1988).

Intersex triploid chickens have been detected each year in our CSIRO Synthetic layer strains at low frequencies $(0.1-0.5 \%)$. Selection for triploidy in one of the Synthetic lines has produced a unique line of chickens that have up to $20 \%$ triploid embryos and $8-12 \%$ hatched triploids each generation. The line also has a low frequency of live haploid-diploid and diploid-triploid chickens (Thorne et al, 1987). Selection line females ovulate a high incidence of diploid ova from errors at both meiosis I and II. Triploids are then produced after fertilization by normal haploid spermatozoa. The tendency to produce diploid ova is genetically determined (Thorne et al, 1980). This paper presents details of the incidence and sex ratio of triploid embryos analyzed in 4 generations of selection for triploidy and the other types of chromosomal abnormalities observed in early embryos.

The high triploid line was developed from a Synthetic layer selection strain that originated from a White Leghorn $\times$ Australorp crossbred flock (Thorne et $a l, 1987)$. The first generation of the line was formed by inbreeding close relatives of intersex triploids (Thorne et al, 1980). The second generation was also produced from inbred matings but thereafter the line has been reproduced at yearly intervals by outbred matings among selected parents. Females of generations 1, 3, 4 and 5 were selected as breeders if they produced one or more triploid embryos out of 20 embryos karyotyped at 3 days of incubation.

To karyotype embryos, eggs were incubated for $72 \mathrm{~h}$, then $0.1 \mathrm{ml}$ of $0.1 \%$ vinblastine sulfate (Lilly) was injected into the air cell. After 2 more hours of incubation, the eggs were opened and classified as either: 1) normal embryos, 
2) slow embryos, 3) dead embryos or 4) membrane development only. Embryonic malformations were recorded if present. Chromosome preparations were made using the technique of Shoffner et al (1967). On every slide, 5 clear metaphase cells were counted and up to 30 metaphases if chromosome abnormalities were present. Interphase nucleoli were counted to confirm the ploidy level. As the chicken has only one nucleolus per haploid chromosome set (Auer et al, 1987), diploid embryos will have 1-2 nucleoli, triploids 1-3 nucleoli, etc.

Chromosome abnormalities were found in 390 embryos out of a total 2936 early chick embryos analyzed in 4 generations of selection for triploidy (table I). They included 207 triploids and another 28 presumptive triploids, each with 1-3 nucleoli, but unclear chromosomes. Three triploids had aneuploid cell lines (1 3AZWW-2; 1 $3 A Z Z Z-2 ; 13 \mathrm{AZZW}+3$ ). The remaining 155 abnormal embryos included 47 haploids (all 1AZ), 1 tetraploid (4AZZWW) and 4 pentaploids (all 5AZZZWW). Another 34 embryos had abnormal nucleoli but unclear polyploid chromosomes including 20 embryos with 1-5 nucleoli, 9 with 1-4 nucleoli and 5 with 1-6 nucleoli.

Table I. Chromosome abnormalities in embryos from tested females in 4 generations of selection for triploidy.

\begin{tabular}{lcccrr}
\hline \multirow{2}{*}{ Abnormality } & \multicolumn{4}{c}{ Hen generation } & Total \\
\cline { 2 - 5 } & $S 1$ & $S 3$ & $S 4$ & $S 5$ & \\
\hline $1 n$ & 8 & 6 & 15 & 18 & 47 \\
$1 n / 2 n$ & 4 & 8 & 16 & 15 & 43 \\
$1 n / 2 n / 3 n$ & - & 1 & - & 3 & 4 \\
$1 n / 2 n / 4 n / \mathrm{HP}^{\mathrm{n}}$ & - & 1 & - & - & 1 \\
$1 n / 3 n$ & 1 & 1 & - & 1 & 3 \\
$1 n / 3 n / 5 n$ & - & - & - & 1 & 1 \\
$2 n / 3 n$ & - & 4 & 1 & - & 5 \\
$2 n / \mathrm{HP}$ & - & - & - & 2 & 2 \\
$2 n / 4 n$ & - & 2 & 1 & 3 & 6 \\
$2 n / 4 n / 8 n$ & - & - & 2 & - & 2 \\
$3 n$ & 23 & 41 & 42 & 101 & 207 \\
$1-3$ NUC & 3 & 6 & 2 & 17 & 28 \\
$4 n$ & - & - & - & 1 & 1 \\
$5 n$ & - & 1 & - & 3 & 4 \\
$1-5$ NUC & 1 & 3 & 1 & 15 & 20 \\
ABN.NUC & - & 2 & 1 & 10 & 14 \\
Other & - & - & 2 & - & 2 \\
Total abnormal & 41 & 76 & 83 & 190 & 390 \\
Total analyzed & 636 & 698 & 810 & 792 & 2936 \\
\hline
\end{tabular}

${ }^{\text {a }}$ HP: high ploidy; NUC: nucleoli; ABN.NUC: abnormal nucleoli.

Haploid mosaics included 43 haploid-diploids (29 Z/ZZ, 14 Z/ZW), 4 haploiddiploid-triploids $(2 \mathrm{Z} / \mathrm{ZZ} / \mathrm{ZZW} ; 1 \mathrm{Z} / \mathrm{ZZ} / \mathrm{ZZZ} ; 1 \mathrm{Z} / \mathrm{ZW} / \mathrm{ZWW})$ and 3 haploidtriploids $(1 \mathrm{Z} / \mathrm{ZWW} ; 2 \mathrm{Z} / \mathrm{ZZZ})$. Two other complex mosaics included a haploid- 
triploid-pentaploid (Z/ZZW/ZZZWW) and a haploid-diploid-tetraploid embryo with higher ploidies (Z/ZZ/ZZZZ/HP).

Diploid-polyploid mosaics included 5 diploid-triploids (4 ZZ/ZZZ; 1 ZW/ZWW), 6 diploid-tetraploids (4 ZW/ZZWW; 2 ZZ/ZZZZ), 2 diploid-tetraploid-octaploids (both $\mathrm{ZW} / \mathrm{ZZWW} / 8 n$ ) and 2 diploid-high ploidies. Two other abnormalities included a trisomic embryo $(2 \mathrm{AZW}+2)$ and a diploid embryo with a Robertsonian translocation involving chromosomes 3 and 4 .

Most of the abnormalities observed in our data have been reported by others (reviewed in Fechheimer, 1981). The chicken appears to have errors of meiosis, fertilization and early cleavage that can produce complex chromosomal abnormalities. In general, the haploid cells of haploid mosaics are derived from mitotic divisions of supernumerary spermatozoa (Fechheimer, 1981), while the diploid-polyploid mosaics $(e g, 2 n / 4 n, 2 n / 4 n / 8 n)$ are thought to result from failure of cytokinesis in early cleavage divisions causing doubling of some cells. The diploid-triploid mosaics, however, have been postulated to arise from binucleated oocytes with meiotic errors in one nucleus (Fechheimer, 1981). The $3 n$ and $5 n$ cells of the $1 n / 3 n / 5 n$ embryo may have been derived from a binucleate oocyte with meiotic errors in both nuclei.

Triploids may arise androgenetically from the union of a haploid ovum with a diploid sperm or 2 spermatozoa (dispermy), or gynogenetically from the union of a diploid ovum with a haploid sperm. Diploid gametes may be produced by chromosomal non-disjunction at meiosis I or II and also in females after suppression of polar body extrusion or by re-entry of a polar body. The ZWW triploids can only be derived from females, while ZZW and ZZZ triploids can arise from either maternal or paternal errors. The sex ratio of triploid embryos (table II) supports a gynogenetic origin. The sex ratios of the 4 generations were not significantly different $(P>0.5)$, so the data were pooled. They show a ratio of ZWW:ZZW:ZZZ triploids which is not significantly different $(P>0.1)$ from the 1:2:1 ratio expected after fertilization of WW, ZW or ZZ diploid ova if MI and MII failed with equal frequency in females. It appears unikely that any of the ZZW or ZZZ triploids are androgenetic in origin. In mice, the LT/Sv strain produces over $30 \%$ triploid embryos because females spontaneously ovulate a high incidence of diploid primary oocytes (O'Neill and Kaufman, 1987).

Table II. Sex ratio of triploid embryos.

\begin{tabular}{ccccc}
\hline Hen generation & $3 A Z W W$ & $3 A Z Z W$ & $3 A Z Z Z$ & Total \\
\hline S1 & 6 & 11 & 6 & 23 \\
S3 & 12 & 20 & 9 & 41 \\
S4 & 7 & 24 & 11 & 42 \\
S5 & 16 & 59 & 26 & 101 \\
Total & 41 & 114 & 52 & 207 \\
\hline
\end{tabular}

Non-disjunction at metaphase of MI and MII is thought to be a possible cause of diploid ova in females. This is supported by the observation that haploid (1AZ) and pentaploid (5AZZZWW) embryos (and unkaryotyped embryos with 15 nucleoli) tended to occur in hens with triploid embryos. The haploids may be 
androgenetic resulting from fertilization of a nulloid polar body or ovum in which the chromosomes have been lost by non-disjunction. The pentaploids may result from fertilization of a ZZWW tetraploid ovum formed by non-disjunction at both MI and MII.

The development of triploid embryos at 3 days of incubation supports the observation that ZWW is lethal (table III). Only $22 \%$ were alive, but all of these were retarded or malformed and were not expected to survive. In comparison, $50 \%$ or more of ZZW and ZZZ triploids were normally developed embryos. Membranes lacking an embryo accounted for almost $80 \%$ of presumptive triploids showing 13 nucleoli. Lack of mitoses in this tissue made karyotyping difficult. Among the malformations observed in triploid embryos, microphthalmia was the most common. The response to selection for triploidy was rapid (table IV). The proportion of tested females producing triploid embryos was $82.0 \%$ by the S5 generation and the incidence of triploid embryos reached $20.8 \%$. The genetic basis of the tendency for diploid ova is currently being studied. The data suggest that there may be a genetically mediated susceptibility to non-disjunction in selection line females which is responsible for the high incidence of diploid ova.

Table III. Development of triploid embryos at 3 days of incubation.

\begin{tabular}{lcccrr}
\hline & Normal embryo & Slow embryo & Dead embryo & Membrane & Total \\
\hline 1-3NUC & $4(2)^{\text {a }}$ & $1(1)$ & 1 & 22 & 28 \\
3AZWW & $1(1)$ & $8(4)$ & $19(10)$ & 13 & 41 \\
3AZZW & $86(11)$ & $9(2)$ & $12(7)$ & 7 & 114 \\
3 AZZZ & $32(5)$ & $5(2)$ & $5(5)$ & 10 & 52 \\
\hline
\end{tabular}

a Parentheses indicate embryos with malformations.

Table IV. Proportion of tested hens with triploid embryos and incidence of triploidy among those hens.

\begin{tabular}{lcccc}
\hline & \multicolumn{4}{c}{ Hen generation } \\
\cline { 2 - 5 } & $S 1$ & $S 3$ & $S 4$ & $S 5$ \\
\hline \% hens with $3 n$ embryos & 40.6 & 58.3 & 54.5 & 82.0 \\
$\% 3 n$ & 11.4 & 15.8 & 12.1 & 20.8 \\
\hline
\end{tabular}

\section{REFERENCES}

Auer H, Mayr B, Lambrou M, Schleger W (1987) An extended chicken karyotype, including the NOR chromosome. Cytogenet Cell Genet 45, 218-221.

de Boer LEM, de Groen TAG, Frankenhuis MT, Zonneveld AJ, Sallevelt J, Belterman RHR (1984) Triploidy in Gallus domesticus embryos, hatchlings and adult intersex chickens. Genetica $65,83-87$

Fechheimer NS (1981) Origins of heteroploidy in chicken embryos. Poult Sci 60, 1365-1371 
O'Neill GT, Kaufman MH (1987) Ovulation and fertilization of primary and secondary oocytes in LT/Sv strain mice. Gametes Res 18, 27-36

Shoffner RN, Krishan A, Haiden GJ, Bammi R, Otis J (1967) Avian chromosome methodology. Poult Sci 46, 333-344

Thorne MH, Sheldon BL, Bobr LW (1980) Preliminary genetic analysis of an apparently inherited triploid intersex condition in the domestic fowl. Proceedings of the South Pacific Poultry Science Convention, Auckland, 65-70

Thorne MH, Collins RK, Sheldon BL (1987) Live haploid-diploid and other unusual mosaic chickens (Gallus domesticus). Cytogenet Cell Genet 45, 21-25

Thorne MH, Collins RK, Sheldon BL, Bobr LW (1988) Morphology of the gonads and reproductive ducts of triploid chickens. Proceedings of the 18th World Poultry Congress, Nagoya, 525-526 